\title{
La Tecnología como Posible Solución a los Problemas Educativos Contemporáneos
}

\author{
Torres-Barreto, Martha Liliana ${ }^{1\left(^{*}\right)}$; Acosta Medina, Julieth Katherin ${ }^{1} \&$ \\ Paba Medina, Maira Camila ${ }^{1}$
}

${ }^{1}$ Universidad Industrial de Santander,

Facultad de Ingenierías Fisicomecánicas, Colombia, Bucaramanga.

\section{RESUMEN}

La educación contemporánea se enfrenta a múltiples problemas tales como la baja motivación de los estudiantes, las altas tasas de deserción o los elevados índices de pobreza de aprendizaje. Estos problemas se han incrementado en el contexto actual, debido a la pandemia del Covid-19. En la lucha contra estas problemáticas surgen las tecnologías educativas como posibles alternativas de solución. Por ello, en el presente artículo se presenta el trabajo desarrollado por un grupo de investigadores colombianos, los cuales aplican el "pensamiento de diseño" para construir una solución disruptiva que contribuya a solucionar algunos de los problemas de los estudiantes y docentes de la educación digital contemporánea. Con el desarrollo de este proyecto, se encuentra que tendencias educativas como la gamificación pueden favorecer en el aumento de la motivación de los estudiantes gracias al uso de elementos de los juegos. Igualmente, los hallazgos indican que la inteligencia artificial puede contribuir en la detección temprana de los alumnos que se encuentran en riesgo de abandonar los cursos, mejorando así el proceso de seguimiento y acompañamiento hacia ellos.

Palabras clave: Educación contemporánea; Gamificación; Inteligencia artificial;Pensamiento de diseño; Problemáticas educativas

\section{Technology as a Possible Solution to Contemporary Educational Problems}

\section{ABSTRACT}

Contemporary education faces multiple problems such as low student motivation, high dropout rates, or high rates of learning poverty. These problems have increased in the current context due to the Covid-19 pandemic. In the fight against these problems, educational technologies emerge as possible alternative solutions. Therefore, this article presents the work developed by a group of Colombian researchers who apply "design thinking" to build a disruptive solution that helps solve some of the problems of students and teachers of digital education contemporary. With the development of this project, it is found that educational trends such as gamification can favor the increase in student motivation thanks to the use of game elements. Likewise, the findings indicate that artificial intelligence can contribute to the early detection of students who are at risk of dropping out of courses, thus improving the process of monitoring and accompanying them.

Keywords: Contemporary education; Gamification; Artificial intelligence; Design thinking; Educational problems 


\section{INTRODUCCIÓN}

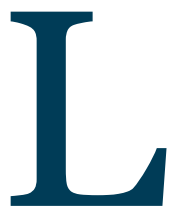

os problemas en la educación se han incrementado notablemente con la aparición del Covid-19, sin embargo, antes de esta pandemia el mundo ya enfrentaba una crisis de aprendizajes. Para el año 2019, 258 millones de niños y jóvenes en edad escolar estaban fuera de la escuela, incluso aquellos que seguían en el sistema educativo aprendían poco, ejemplo de ello, la tasa de pobreza de aprendizajes para la educación primaria en los países de ingreso bajo y mediano era del $53 \%$, lo que significaba que más de la mitad de los estudiantes no podían leer y comprender un relato sencillo adecuado para su edad (Banco Mundial, 2020).

La educación superior no es la excepción en estos problemas, para el caso de Colombia las tasas de abandono en los programas de formación virtual se acercan al 60\% (Ministerio de Educación Nacional de Colombia, 2017). Además, la baja motivación en los estudiantes universitarios es un factor común para varias carreras en muchos países, este elemento es una de las causas del escaso aprendizaje por los alumnos, lo que plantea la necesidad de establecer estrategias que generen motivación intrínseca (Espinoza Freire et al., 2020). Por esto, la desmotivación progresiva de los estudiantes es una las barreras habituales que se detectan cuando se llevan a cabo iniciativas en la educación digital (Gutiérrez Priego \& García Peralta, 2016), modalidad de aprendizaje indispensable en el contexto actual, inmerso en confinamientos y distanciamiento social (Mendiola et al., 2020).

Sin embargo, aparece en el panorama una posible solución a estos problemas, la promoción de estrategias tecnológicas en las aulas. Dentro de estas iniciativas, la gamificación y la inteligencia artificial surgen como herramientas prometedoras para los procesos digitales de enseñanza y aprendizaje del siglo XXI, pues permiten mejorar los entornos de aprendizaje para hacerlos más agradables aumentando la motivación, el compromiso y la retención del conocimiento (Filippou et al., 2018).

Como consecuencia, surge la necesidad de desarroIlar una solución disruptiva que emplee tecnologías educativas y contribuya a solucionar algunos de los problemas que enfrenta la educación digital contemporánea. Por esto, un grupo de investigadores colombianos aplicó el método de design thinking con el objetivo de analizar, estudiar, diseñar y desarrollar un proyecto educativo enfocado en satisfacer las necesidades de estudiantes y docentes de educación virtual. En el presente artículo se explican las bases teóricas, el enfoque metodológico y los resultados de este proyecto.

\section{MARCO TEÓRICO}

\subsection{Problemas de la educación contemporánea}

Dentro de los principales problemas a los que se enfrenta la educación en todas sus modalidades y niveles educativos es la deserción estudiantil (Duque González et al., 2020). Este fenómeno hace referencia al abandono escolar de los estudiantes causados por una combinación de diversos factores; se puede clasificar en deserción precoz la cual ocurre antes de la matricula, temprana o en primeros periodos y tardía que se presenta en últimos semestres (Estévez et al., 2015). Asimismo, esta situación se ha convertido en los últimos años en una problemática escolar, principalmente para los países de ingresos bajos o medios, donde la pobreza del aprendizaje, un indicador que refleja las bajas capacidades académicas y de comprensión que poseen los estudiantes en los diferentes niveles educativos, presenta porcentajes muy altos (World Bank, 2019).

Igualmente, otro de los desafíos educativos, princi- 
palmente desde la virtualidad, es la baja motivación de los estudiantes, un estado de comportamiento en el que las personas no tienen razones para guiar sus acciones hacia el aprendizaje, perdiendo el entusiasmo, el interés, la energía y la disposición (Berridi Ramírez et al., 2017). Además, aunque en la mayoría de los casos la motivación es alta al inicio de los cursos, esta va decayendo conforme avanzan las temáticas y aumenta la complejidad (Vidal \& Olivares, 2018).

\subsection{Tecnologías educativas}

Las tecnologías educativas hacen referencia a la incorporación de procesos, recursos o herramientas tecnológicas en los procesos de enseñanza y aprendizaje (Torres Cañizález \& Cobo Beltrán, 2017). Estas tecnologías pueden derivar en un aprendizaje experiencial, inmersivo y personalizado, fundamental para los estudiantes contemporáneos, una generación rodeada por elementos digitales y juegos en línea, quienes requieren nuevos enfoques pedagógicos (Yturralde Villagómez et al., 2020).

En este sentido, para lograr experiencias de aprendizaje inmersivas y vivenciales es posible usar tecnologías asociadas como la realidad virtual y la realidad mixta, las cuales permiten crear entornos con apariencia real en escenarios totalmente virtuales (Organización de Estados Iberoamericanos, 2018); sin embargo, estas requieren dispositivos tecnológicos o artefactos como gafas, cascos, guantes y trajes especiales, por lo que sólo se pueden utilizar en cursos presenciales (León Rodriguez \& Viña Brito, 2017).

Por otro lado, para alcanzar aprendizajes personalizados con contenidos dinámicos de acuerdo con las necesidades de los usuarios, es posible implementar recursos como la gamificación o la incorporación de elementos propios de los juegos y la inteligencia artificial, siendo estos, recursos que se pueden aplicar en escenarios presenciales y virtuales (Jara \& Ochoa, 2020).

\section{METODOLOGÍA}

La metodología usada en este proyecto se basa en el design thinking, un método escalable que incluye un espectro de actividades de innovación con espíritu de diseño centrado en el hombre. Generalmente, se aplica gradualmente para mejorar las ideas existentes o para crear soluciones disruptivas que satisfagan las necesidades de las personas en formas completamente nuevas (Castillo Vergara et al., 2014). El design thinking permite recuperar el pensamiento innovador en la educación y se compone por 5 etapas: empatizar, definir, idear, prototipar y probar (Brown, 2008).

En la etapa de "empatizar" se busca entender al usuario, sus hábitos, motivaciones, preocupaciones, necesidades y aspiraciones; por ello se aplicaron herramientas investigativas tales como entrevistas, encuestas, focus group y perfiles de usuario (Ortega $\&$ Ceballos, 2015). Por su parte, en la etapa de "definir" se requiere converger toda la información recolectada para encontrar los insights y necesidades de los usuarios, en el contexto de este estudio se emplearon mapas de empatía y journey maps (CastiIlo Vergara et al., 2014).

Igualmente, en la etapa de "idear" se empiezan a crear soluciones para los diferentes problemas encontrados; en esta fase se utilizaron instrumentos tales como el brainstorming o lluvia de ideas y el storyboarding o conjunto de viñetas secuenciales, instrumentos apropiados para el diseño de sistemas interactivos que cambiarán la forma de realizar ciertas tareas (Shah et al., 2020). En relación con la etapa "prototipar", se materializaron las ideas permitiendo visualizar e identificar los puntos clave, aciertos, problemas y condiciones en prototipos digitales (Apel et al., 2020).

Finalmente, en la etapa de "testear" se evalúan y validan los prototipos para perfeccionarlos y convertirlos en soluciones que responda de manera acertada a las necesidades de los usuarios; por esto, en este caso se tuvieron en cuenta los resultados de la ejecución de pruebas controladas con usuario en las que se aplicaron interacciones constructivas, evaluaciones de la experiencia y pruebas de usabilidad (Brown \& Wyatt, 2010). 


\section{RESULTADOS Y/O DISCUSIÓN}

\subsection{Empatizar}

Inicialmente, se aplicaron encuestas y entrevistas a 132 estudiantes y 52 profesores de educación superior en modalidad digital. A partir de la información cualitativa y cuantitativa recolectada se construyeron perfiles de usuarios para estos dos grupos, agentes activos de los procesos de enseñanza y aprendizaje.

Con relación a los estudiantes se identificaron cuatro perfiles que se encuentran resumidos en la Tabla 1. Se encontró además, que la mayoría de ellos tienen edades entre 18 a 24 años y se encuentran trabajando.

\section{Tabla 1. Perfiles estudiantes de educación virtual}

\begin{tabular}{|c|c|}
\hline Perfil & Características \\
\hline $\begin{array}{l}\text { Estudiante } \\
\text { trabajador }\end{array}$ & $\begin{array}{l}\text { Datos sociodemográficos: } 45 \text { años. Casado. Con hijos. } \\
\text { Estrato 4. Gerente General. Estudia un MBA. } \\
\text { Comportamiento: Estudia desde casa en las noches, no } \\
\text { tiene mucho tiempo por lo que le afectan los extensos } \\
\text { trabajos, se siente desactualizado con las plataformas } \\
\text { virtuales de estudio. } \\
\text { Motivaciones y Necesidades: Necesita canales de } \\
\text { comunicación rápidos y plataformas con navegación } \\
\text { intuitiva, quiere certificar su experiencia para obtener un } \\
\text { mejor salario. }\end{array}$ \\
\hline $\begin{array}{l}\text { Estudiante } \\
\text { recién } \\
\text { graduado }\end{array}$ & $\begin{array}{l}\text { Datos sociodemográficos: } 20 \text { años. Soltero. Trabaja con } \\
\text { sus padres en la zona rural. Estudia una tecnología. } \\
\text { Comportamiento: Los problemas familiares afectan el } \\
\text { ritmo de estudio, se le dificulta la comunicación y la } \\
\text { información que le suministra el profesor es insuficiente. } \\
\text { Motivaciones y Necesidades: Necesita canales de } \\
\text { comunicación rápidos, quiere tener una mejor calidad de } \\
\text { vida y las malas notas lo alertan }\end{array}$ \\
\hline $\begin{array}{l}\text { Estudiante } \\
\text { retos } \\
\text { personales }\end{array}$ & $\begin{array}{l}\text { Datos sociodemográficos: } 63 \text { años. Casada. Con hijos } \\
\text { adultos. Estrato } 4 \text {. Pensionada. Estudia un pregrado. } \\
\text { Comportamiento: Tiene mucho tiempo libre, pero se } \\
\text { cansa con facilidad. Es organizada pero olvidadiza. Le } \\
\text { cuesta adaptarse al ritmo de estudio } \\
\text { Motivaciones y Necesidades: Necesita canales de } \\
\text { comunicación fáciles, la motivan los retos y requiere una } \\
\text { plataforma de estudio intuitiva y amigable }\end{array}$ \\
\hline $\begin{array}{l}\text { Estudiante } \\
\text { fantasma }\end{array}$ & $\begin{array}{l}\text { Datos sociodemográficos: No aplica. } \\
\text { Comportamiento: Se matricula a los cursos para cobrar } \\
\text { por hacer actividades para otros estudiantes o para robar } \\
\text { contenido. } \\
\text { Motivaciones y Necesidades: Su mayor motivación es el } \\
\text { dinero. Le interesa crear contactos y vender el programa }\end{array}$ \\
\hline
\end{tabular}

En cuanto a los docentes se encontraron 3 perfiles resumidos en la Tabla 2 y se evidenció que la mayoría de ellos tiene a cargo grandes volúmenes de estudiantes, por lo que se les dificulta realizar un proceso de seguimiento y acompañamiento.

\begin{tabular}{|c|c|}
\hline Perfil & Características \\
\hline $\begin{array}{l}\text { Docente } \\
\text { empático }\end{array}$ & $\begin{array}{l}\text { Datos sociodemográficos: } 45 \text { años. Casada. Con hijos. } \\
\text { Estrato 5. Magister. Profesora virtual y presencial } \\
\text { Comportamiento: Se preocupa por sus estudiantes y está } \\
\text { pendiente de ellos, deja trabajos dinámicos y variados. } \\
\text { Motivaciones y Necesidades: Necesita canales de } \\
\text { comunicación efectivos y herramientas para motivar a sus } \\
\text { estudiantes. }\end{array}$ \\
\hline $\begin{array}{c}\text { Docente } \\
\text { normativo }\end{array}$ & $\begin{array}{l}\text { Datos sociodemográficos: } 32 \text { años. Casado. Sin hijos. Con } \\
\text { doctorado. Estrato 4. Profesor virtual y presencial. } \\
\text { Comportamiento: Se limita a dictar sus clases, solo } \\
\text { responde dudas por correo, no está pendiente de sus } \\
\text { estudiantes. } \\
\text { Motivaciones y Necesidades: Necesita estudiantes } \\
\text { organizados y con autonomía. Afirma que el seguimiento } \\
\text { lo debe hacer la plataforma y no él. }\end{array}$ \\
\hline $\begin{array}{c}\text { Profesor } \\
\text { activo }\end{array}$ & $\begin{array}{l}\text { Datos sociodemográficos: } 42 \text { años. Casado. Con hijos. } \\
\text { Estrato } 3 \text {. Profesional con } 23 \text { años de experiencia. Profesor } \\
\text { virtual. } \\
\text { Comportamiento: Le gusta generar confianza con sus } \\
\text { estudiantes, planea sus clases con anterioridad. } \\
\text { Motivaciones y Necesidades: Calificar se le convierte en } \\
\text { un proceso tedioso por los grandes volúmenes de trabajos. } \\
\text { No sabe cómo organizar a sus estudiantes. Quiere } \\
\text { personalizar sus clases. }\end{array}$ \\
\hline
\end{tabular}

\subsection{Definir}

IDespués de desarrollar los perfiles y escoger como usuario objetivo al perfil de estudiante recién graduado debido a la alta tasa de alumnos que este representa, se procedió a desarrollar mapas de empatía y journey maps para este tipo de usuario, en los que se identificaron los siguientes pain points o experiencias negativas:

- Las notificaciones de las actividades no llegaban al correo ni a ningún otro medio.

- El sistema no contaba con notificaciones sobre las calificaciones o retroalimentaciones del profesor y compañeros.

- Había que recorrer un largo camino en la plataforma para encontrar las actividades y las notas.

- La plataforma desmotivaba la ejecución de las actividades académicas y no reforzaba el desarrollo de capacidades necesarias para la digitalidad.

- El profesor tardaba en responder las preguntas o no las respondía. Debido a que las preguntas estaban mal formuladas y no eran claras, o bien, a la poca efectividad de los canales de comunicación. 


\subsection{Idear}

Con base en los hallazgos encontrados en la etapa anterior, se plantea una solución compuesta por dos elementos. El primero dirigido a aumentar la motivación de los estudiantes y a reforzar los procesos de enseñanza y aprendizaje y el segundo enfocado en optimizar los canales de comunicación y apoyar a los docentes en el seguimiento de sus alumnos. Para la ideación del primer elemento se aplicó la herramienta de storyboarding, la cual consiste en un conjunto de imágenes o dibujos dispuestos de forma secuencial (Ver Figura 1).

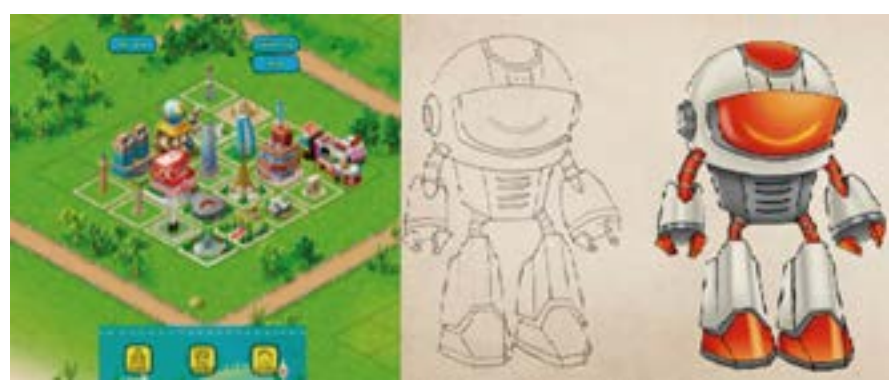

Figura 1. Storyboarding- Primer elemento

Cabe resaltar, que en este elemento se aplicaron los principios de la gamificación, tendencia educativa que utiliza componentes de los juegos para dinamizar los ambientes de aprendizaje, fomentar el desarrollo de habilidades como autonomía u organización y facilitar la adquisición de conocimiento (Acosta-Medina et al., 2020).

A partir de este ejercicio, se plantea el desarrollo de una herramienta gamificada motivacional enfocada en reforzar el aprendizaje sobre competencias ciudadanas, habilidades transversales a cualquier curso o carrera. Esta temática fue elegida, debido a la importancia que esta tiene en el contexto actual, pues favorece la solución de conflictos y la convivencia pacífica. Además, que son competencias útiles para cualquier programa de formación (Paba Medina et al., 2020).

Por otro lado, para la ideación del segundo elemento se utilizó el brainstorming o la lluvia de ideas, aplicada durante 5 sesiones en las que participaron equipos interdisciplinarios conformados por docentes, ingenieros, administradores, diseñadores, comunicadores, empresarios y estudiantes. Los resultados de este proceso indicaron la necesidad de desarrollar una plataforma de contactabilidad basada en inteligencia artificial, que mejorara la efectividad y rapidez de las comunicaciones entre los estudiantes y sus ambientes virtuales de aprendizaje. Adicionalmente, se plantea que esta plataforma incluya una interfaz interactiva que le muestre a los docentes el nivel de riesgo de deserción en el que se encuentran sus estudiantes, facilitándole el proceso de seguimiento y comunicación. Dicho riesgo de deserción se obtiene a través de modelos predictivos que aplican algoritmos de machine learning.

\subsection{Prototipar}

En el contexto de este estudio, en la cuarta etapa se desarrollan prototipos de interacción de bajo nivel para comprobar los esquemas de navegación y posteriormente se construyen prototipos de alta fidelidad gráfica e interacción para evaluar la comunicabilidad, usabilidad y desempeño del usuario.

Para el prototipado de la herramienta gamificada se elige el motor multiplataforma Unity 3D, debido a que este desarrollador de videojuegos admite conexiones a través de servicios web, permite el desarrollo por capas gráficas, facilita controlar los factores de comportamiento desde una consola de programación, tiene la posibilidad de compilar el producto estándar en html5 y brinda un soporte óptimo para en la curva de desarrollo.

En total se generaron dos prototipos de la herramienta. El primer prototipo alcanzó el desarrollo de funcionalidades básicas para implementar la mecánica del videojuego en el primer nivel e incluía: recolección de recursos, construcción de edificaciones, avance en el nivel de juego, eliminación de edificaciones y resolución de los tres tipos de misiones de educación cívica. Para el segundo prototipo se profundizo en la jugabilidad y se replicaron las funcionalidades del primer nivel a los siete niveles restantes, incorporando el gasto de recursos por el funcionamiento de la ciudad, los avatares y nuevos mapas de la ciudad.

Por su parte, para el prototipado de la plataforma de contactabilidad se utiliza el lenguaje de programación Phyton para el tratamiento de datos y cálculos complejos relacionados con el modelo de predic- 
ción. Además, se usan componentes backend para el procesamiento de información y PHP para soportar los procesos transaccionales, así como los eventos de creación, lectura y actualización de registros. Adicionalmente,

la plataforma hará uso de MongoDB por sus características open-source y de NoSQL por su alta asociabilidad con el análisis de datos en tiempo real, usando el servicio de control de versiones Codecommit provisto por Amazon Web Services, el cual permite tener un esquema de trabajo colaborativo en el que cada integrante puede confirmar, bifurcar y combinar el código para mantener el control sobre el software de forma sencilla.

Específicamente, el modelo machine learning se construye con el componente xgboost, el cual analiza los datos de la semana 1 a la semana 4 del curso virtual y durante la semana 4 ya puede predecir si el estudiante esta desmotivado o perdió el interés por las actividades del curso. Con relación a la interfaz interactiva, esta se construyó con una navegación intuitiva y de fácil manejo, que permite a los administradores revisar el estado de todos los estudiantes de la institución educativa, verificar los mensajes que han sido enviados y enviar un reporte grafico a cada docente en el que se indica el nivel de riesgo de deserción en el que se encuentran los estudiantes de sus cursos.

\subsection{Probar}

Para la evaluación de los prototipos se realizaron pruebas experimentales controladas con usuarios de manera remota, en estas los estudiantes ejecutaron diferentes actividades en los productos y luego respondieron un cuestionario de percepciones.

En la evaluación del primer prototipo de la herramienta gamificada se encontró que la apariencia percibida no coincidía con la visión del misma, lo que hizo necesario la incorporación de mejoras gráficas en el aspecto futurista de las edificaciones de la ciudad, pues el objetivo era presentar una ciudad futurista. Sin embargo, se encontró que la estrategia gamificada tenía una usabilidad integrada aceptable superior al $70 \%$ y la experiencia de juego obtuvo una valoración de 1,11 mostrando percepciones positivas con respecto a la utilidad de la experiencia, diversión, creatividad y estilo. De igual forma, la calidad estética obtuvo en promedio 5,76 puntos considerando pertinente la selección del color y valorando la sencillez y el orden de los elementos de la interfaz de usuario.

Teniendo en cuenta estos resultados se llega al prototipo final de la herramienta digital gamificada, la cual tiene formato de videojuego y mecánica predominante de construcción. En esta los usuarios asumen el rol de alcaldes, administran recursos públicos y resuelven diferentes misiones para recibir monedas y construir una nueva ciudad fundamentada en las competencias ciudadanas. Actualmente, se están realizando las pruebas al prototipo final en las que se están midiendo indicadores de aprendizaje de competencias ciudadanas (pre-test, post-test), engagement y gamificación.

De otra forma, para la validación del modelo machine learning se ejecutaron seis (6) corridas ajustando los datos que se traen de la plataforma de aprendizaje y llegando finalmente a una precisión del 94,4\%, entrenando el modelo con más de 7000 registros de estudiantes. Adicionalmente, al aplicar una interacción constructiva con la plataforma de contactabilidad, se encontró que mientras los usuarios interactuaban con el prototipo y desarrollaban una serie de actividades preestablecidas, era fundamental considerar los niveles de información que recibiera cada docente, pues este último solo necesita conocer el análisis cognitivo y las interacciones sociales de los estudiantes de sus cursos para revisar qué aspectos ocurren antes de la deserción del estudiante y sugerir una serie de acciones de mejora. 


\section{CONCLUSIONES}

Tecnologías como la inteligencia artificial no solo permiten realizar diferentes predicciones relacionadas con aspectos académicos de los estudiantes, sino que también facilitan descubrir patrones, asociaciones, cambios, anomalías y estructuras más significativas permitiendo tomar acciones que mejoren los procesos de enseñanza y aprendizaje.

De igual manera, tendencias como la gamificación o el uso de elementos de los juegos emergen desde la industria del desarrollo web, como unas de las soluciones al problema de la motivación de los estudiantes, en tanto que hacen las actividades más activas y participativas, mejorando la experiencia e interacción con conceptos propios de las materias, pero en entornos más interactivos. Además, estas estrategias sirven de apoyo a los programas de educación presencial y virtual, ya que no requiere la presencia física del individuo.

Para futuras investigaciones se invita a seguir estudiando las contribuciones de tecnologías como la gamificación o la inteligencia artificial en contextos educativos, pues estas representan una oportunidad para lograr experiencias de aprendizaje significativas, interesantes, atractivas y personalizadas. Además, se sugiere seguir una línea de investigación con un espectro más amplio de tecnologías educativas, considerando proyectos que incluyan realidad virtual, robótica educativa o realidad mixta.

\section{AGRADECIMIENTOS}

Agradecimiento especial a la Universidad Industrial de Santander y al Sistema General de Regalías fondos de CTel de la Gobernación de Antioquia administrados a través del Patrimonio Autónomo Fondo Nacional de Financiamiento para la Ciencia, la Tecnología y la Innovación Francisco José de Caldas -MINCIENCIAS-, por el apoyo financiero recibido para la realización de este proyecto. 


\section{REFERENCIAS}

Acosta-Medina, J. K., Torres-Barreto, M. L., \& Alvarez-Melgarejo, M. (2020). Literature mapping about gamification in the teaching and learning processes. Revista ESPACIOS, 41(11), 26. https://www.revistaespacios.com/a20v41n11/ a20v41n11p26.pdf

Apel, A., Hull, P., Owczarek, S., \& Singer, W. (2018). Transforming the Enrollment Experience Using Design Thinking. College and University, 93(1), 45-50.

Banco Mundial. (2020). COVID-19 : Impacto en la Educación y respuesta de Política Pública. http://documents1.worldbank.org/curated/en/804001590734163932/pdf/The-COVID-19 -Pandemic-Shocks-to-Education-and-Policy-Res ponses.pdf

Berridi Ramírez, R., \& Martínez Guerrero, J. I. (2017). Estrategias de autorregulación en contextos virtuales de aprendizaje. Perfiles educativos, 39(156), 89-102.

Brown, T. (2008). Design Thinking - Design Básico. Harvard Business Review, 1-10.

Brown, T., \& Wyatt, J. (2010). Design thinking for social innovation. Development Outreach, 12(1), 29-43.

Castillo Vergara, M., Álvarez Marín, A., \& Cabana Villca, R. (2014). Design thinking: Cómo guiar a estudiantes, emprendedores y empresarios en su aplicación. Ingeniería Industrial, 35(3), 301-311.

Duque González, C. A., Leal Martínez, D. C., Cuesta García, J. F., González Romero, O. E., \& Ardila Ochoa, S. V. (2020). Generación de estrategias en la innovación de la educación con el fin de mitigar la deserción universitaria.

Espinoza Freire, E. E., Villacres Arias, G. E., \& Granda Ayabaca, D. M. (2020). Influencia de las didácticas tecnológicas en el desarrollo del aprendizaje de los estudiantes. Revista Metropolitana de Ciencias Aplicadas, 3(3), 63-70.

Estévez, J. A., Castro-Martínez, J., \& Granobles, H. R. (2015). La educación virtual en Colombia: exposición de modelos de deserción. Revista de Innovación Educatival, 7(1), 94-107.
Filippou, J., Cheong, C., \& Cheong, F. (2018). A model to investigate preference for use of gamification in a learning activity. Australasian Journal of Information Systems, 22, 1-23. https://doi.org/10.3127/ajis.v22i0.1397

Gutiérrez Priego, R., \& García Peralta, A. (2016). ¿Cómo mejorar la calidad, la motivación y el compromiso estudiantil en la educación virtual? Campus Virtuales, 5(1), 74-82.

Jara, I., \& Ochoa, J. M. (2020). Usos y efectos de la inteligencia artificial en educación.

León Rodriguez, G. de la C., \& Viña Brito, S. M. (2017). La inteligencia artificial en la educacion superior. Oportunidades y amenazas. INNOVA Research Journal, 2(8.1), 412-422.

https://doi.org/10.33890/innova.v2.n8.1.2017.399

Mendiola, M. S., Hernández, A., Torres, R., Carrasco, M., Romo, A., Mario, A., \& Cazales, V. (2020). Retos educativos durante la pandemia de COVID-19: una encuesta a profesores de la UNAM. Revista Digital Universitaria, 21(3).

Ministerio de Educación Nacional de Colombia. (2017). Reporte sobre deserción y graduación en educación superior.

Organización de Estados Iberoamericanos. (2018). Neurodidáctica en el aula: Transformando la educación. Revista Iberoamericana de Educación, 78(1).

Ortega, M. S., \& Ceballos, P. B. (2015). Design thinking: Lidera el presente. Crea el futuro. ESIC Editorial.

Paba Medina, M. C., Acosta Medina, J. K., \& Torres Barreto, M. L. (2020, June 2). Design of a gamified tool for the development of citizenship competencies. 6th International Conference on Higher Education Advances (HEAd'20). https://doi.org/10.4995/HEAd20.2020.11136

Shah, J. J., Vargas-Hernandez, N. O. E., Summers, J. D., \& Kulkarni, S. (2001). Collaborative Sketching (C-Sketch)-An idea generation technique for engineering design. The Journal of Creative Behavior, 35(3), 168-198.

Torres Cañizález, P. C., \& Cobo Beltrán, J. K. (2017). Tecnología educativa y su papel en el logro de los fines de la educación. Educere, 21(68), 31-40. 
Vidal, L. A., \& Olivares, J. H. (2018). Factores psicosociales y su influencia en la desmotivación escolar. In LOS PROBLEMAS DE LA ENSEÑANZA DE LA HISTORIA, GEOGRAFÍA Y CIENCIAS SOCIALES EN LA ENSEÑANZA MEDIA (pp. 19-31).

World Bank. (2019). Ending Learning Poverty: What Will It Take? http://hdl.handle.net/10986/32553

Yturralde Villagómez, J. C., Trejo Alarcón, J. E., Chiquito Peñaranda, D. A., \& Rodríguez López, W. A. (2020). El empleo de las aplicaciones de las tecnologías de la información y las comunicaciones en el proceso enseñanza-aprendizaje en la educación superior. Dilemas Contemporáneos: Educación, Política y Valores, 7. 